\title{
Estimating peak velocity of rapid eye movements from video recordings
}

\author{
J. T. ENRIGHT \\ Scripps Institution of Oceanography, La Jolla, Califormia
}

\begin{abstract}
Although video offers many advantages for recording human eye orientation, it involves such low temporal resolution $(60 \mathrm{~Hz})$ that it seems an unpromising method for evaluating the dynamics of rapid (saccadic) eye movements. This study demonstrates, nevertheless, that such measurements can provide surprisingly reliable estimates of the peak velocity of larger saccades. Simulations of $60-\mathrm{Hz}$ sampling of eye position during idealized saccades provided replicated estimates of "apparent peak velocity." The results indicate that when saccadic amplitude is about $10^{\circ}$ or larger, estimates of peak velocity would on average be biased downward by less than $10 \%$, with standard deviations due to measurement timing of less than 5\%. Experimental data (from recordings of $10^{\circ}$ and $20^{\circ}$ saccades with customized video) demonstrate that these theoretical sources of uncertainty are considerably smaller than the trialto-trial variability in performance of real saccades. Reliability of video recording, however, rapidly deteriorates when saccades become smaller than about $10^{\circ}$.
\end{abstract}

Monitoring of a subject's eye movements is becoming an increasingly common component of various kinds of behavioral research, and for that purpose, a variety of eye-tracking systems based on video are now available (Clarke, 1994). Video has a variety of advantages: It is noninvasive and less expensive than many alternative methods, and it readily permits simultaneous evaluation of pupil size and - given adequate iris landmarks - torsional orientation (i.e., rotation of the eye around the line of sight). Furthermore, replay of a videotape permits repeated visual examination of actual performance, so that subtle artifacts can be detected (e.g., miniature eye blinks that can deflect the eye but do not reach the pupil or lateral margins of the cornea). Nevertheless, video also has its disadvantages. Theoretical analyses of potential artifacts associated with certain aspects of video eye tracking have recently been published (Haslwanter \& Moore, 1995; Moore, Haslwanter, Curthoys, \& Smith, 1996). There is, in addition, a single previously published evaluation of the limitations of a video-based system for estimating critical dynamic properties of saccades, such as peak velocity (Harris, Abramov, \& Hainline, 1984), and that article painted a very discouraging picture. For example, it indicated that peak velocity of $10^{\circ}$ saccades would on average be underestimated by about $40 \%$, with a very large "noise" level; such a conclusion seems intuitively plausible because of the obvious mismatch between the time course of eye dynamics and the $50-$ or $60-\mathrm{Hz}$ sampling rate of video.

This research was supported by Grant IBN93-13038 from the National Science Foundation. Helpful comments and advice were received from Lo Bour, Angelique Hendriks, L. Abel, T. Haslwanter, and two anonymous referees. Correspondence should be addressed to J. T. Enright, Neurobiology Unit 0202, Scripps Institution of Oceanography, La Jolla, CA 92093 (e-mail: jenright@ucsd.edu).
Consider, however, the experimental data presented in Figure 1: estimates of "apparent peak saccadic velocity" plotted against saccadic excursion, for adducting and abducting saccades (toward and away from the midline) with targets separated by $10^{\circ}$ and by $20^{\circ}$. The difference in positions of the two regression lines (derived from calculations that used log-transformed data) is conspicuous; the mean difference in peak velocity for a given excursion is about $9 \%$, or $25 \% \mathrm{sec}$ to $35 \% \mathrm{sec}$, an amount that is highly significant $(p<.001$, evaluated by analysis of variance). The phenomenon involved in those data (abducting saccades of a given excursion being more rapid than adducting) is by no means novel, having been thoroughly documented in several previous publications (e.g., Collewijn, Erkelens, \& Steinman, 1988). The remarkable aspect of the results in Figure 1 is only that the data were derived from video recordings, meaning that each estimate of peak velocity was based on position measurements from a rapidly moving eye, obtained with an extremely coarse sampling rate $(60 \mathrm{~Hz})$. The objective of this article is to evaluate some of the potential problems underlying this methodology and the resulting data. It turns out that the consistency of the measurements shown in Figure 1 is not as surprising as it may seem to be. The data can readily be reconciled with the video monitoring method from which they were derived.

\section{POTENTIAL PROBLEMS WITH VIDEO RECORDING}

The first of three obvious potential problems associated with using video monitoring of eye position to estimate eye velocity during a saccade is the image smear that arises with ordinary video during rapid rotation of the eye. Modern CCD cameras, however, typically incorporate an electronic shutter that can obviate that problem. 


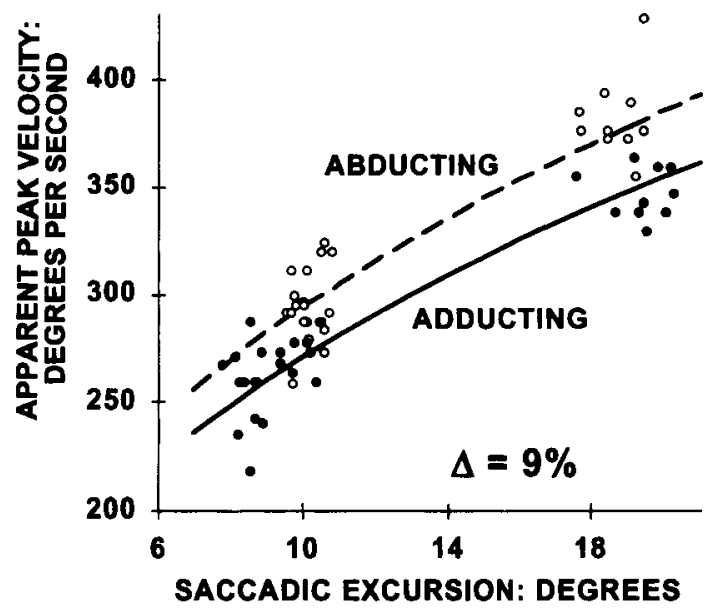

Figure 1. Estimates of apparent peak saccadic velocity for abducting (temporally directed: open circles) and adducting (nasally directed: filled circles) horizontal saccades, together with least squares regression lines based on logarithmically transformed data. Plotted data derived from limbus-margin positions measured on 33-cm monitor (Sony, Model CVM 131), during fieldby-field playback of 60 -Hz video recordings that were made using a CCD camera with a shutter speed of $1 / 500 \mathrm{sec}$ (Cohu Electronics, Model 4915-2000); a zoom lens (Sony, 1:1.8, f12.5, $75 \mathrm{~mm}$, producing an approximately sevenfold magnification on the monitor); and an SVHS video recorder (Panasonic, Model AG 1960)equipment obtainable for about $\$ 3,000$. The $9 \%$ difference in positions of regression lines was highly significant $[F(1,57)=$ $32.2, p<.001]$.

The recordings leading to Figure 1 utilized a moderately priced CCD camera, designed for surveillance, with a shutter speed of $1 / 500 \mathrm{sec}$, meaning that each individual video field provided a crisp, unblurred image even during very rapid eye movements.

The second potential problem involves measurement error, but the imprecision of manual position estimates from these video recordings also turns out to be a less severe problem for such data than one might suspect. Replicated "blind" evaluations of eye-movement recordings (limbus position measured relative to an electronically superimposed image-splitting line), with sevenfold enlargement of the eye on the monitor screen, have shown that the precision of single measurements involves a standard deviation of about 6 arc min (Enright, 1984). Hence, the difference in two sequential measurements, from which velocity might be estimated, would be expected to have a standard deviation, due to measurement imprecision, of about $8.4 \mathrm{arc} \min$ (i.e., $\sqrt{2}$ multiplied by 6 arc $\min$ ). Since the time interval between measurements is $1 / 60 \mathrm{sec}$, the standard deviation of estimates of velocity resulting from random measurement errors would be expected to be about $8.4 \% \mathrm{sec}$. The standard deviations of the single estimates in Figure 1 around the two regression lines were in fact $14 \%$ sec for abduction and $17 \%$ sec adduction, indicating that measurement imprecision would have contributed only $25 \%-35 \%$ of the observed variance.
The third and seemingly insuperable problem associated with video recording is its coarse sampling rate, since true peak velocity is an instantaneous value of a rapidly changing variable. With recording at $60 \mathrm{~Hz}$, individual measurements of position are separated from each other by $16.66 \mathrm{msec}$; the total duration of the saccades of Figure 1 was on the order of $50-80 \mathrm{msec}$, during which velocity accelerated from zero to its maximum and returned again to zero; "apparent peak velocity" has thus been derived from three to no more than five sequential, widely spaced estimates of position during that entire acceleration and deceleration. On the surface of it, therefore, it seems surprising indeed that such a coarse temporal resolution can permit reliable detection of a less-than-10\% difference in average peak velocities between abduction and adduction. This paper describes an elementary simulation study addressing that issue. The influence of coarse temporal resolution on estimates of peak velocity is examined, both in terms of resulting biases and of expected variability of the estimates.

\section{THE SIMULATIONS}

A third-order polynomial (cubic) equation was used as an elementary analytical framework for simulating the time course of saccadic velocity:

$$
V=a t+b t^{2}+c t^{3}
$$

where $t$ represents time after onset of movement and $a$, $b$, and $c$ are parameters to be assigned. Acceleration during a saccade is usually somewhat more rapid than deceleration, meaning that profiles of saccadic velocity typically peak somewhat before midtime of the saccade. The three forms of Equation 1 that have been considered in detail are illustrated in Figure 2, distinguished by whether

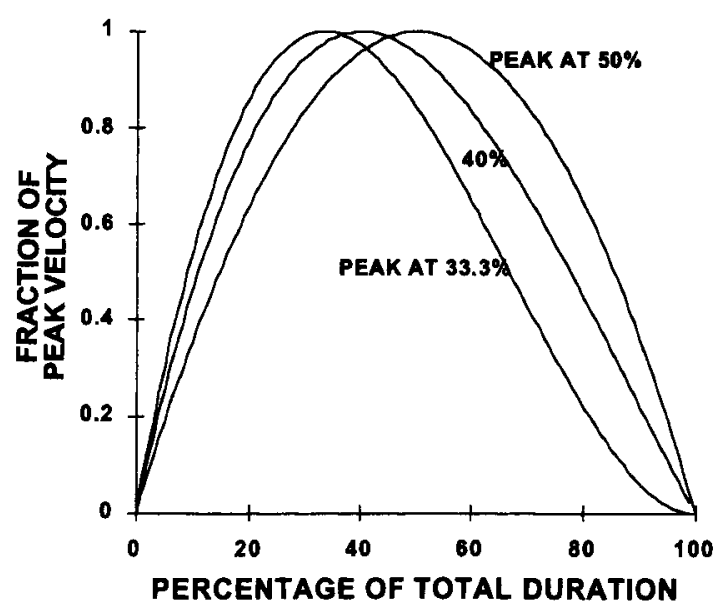

Figure 2. Examples of third-order polynomial equations for velocity as a function of time, $t$, after saccadic onset $\left(V_{t}=a t+\right.$ $b t^{2}+c t^{3}$, with values for $a, b$, and $c$ chosen to produce peak velocity at $33.3 \%, 40 \%$, and $50 \%$ of saccadic duration). When peak velocity occurs at $50 \%$ of duration, $c$ becomes zero and the cubic equation degenerates into a quadratic. 
peak velocity occurred at $33.3 \%, 40 \%$, or $50 \%$ of the total saccadic duration. Figure 3 compares actual velocity profiles from $10^{\circ}$ and $20^{\circ}$ saccades (as measured at $500 \mathrm{~Hz}$ with the best currently available methodology: scleral-search-coil data from Collewijn et al., 1988, Figure 2) together with fitted variants of Equation 1 having peak velocity at $40 \%$ and at $50 \%$ of total duration. (With timing of peak velocity appreciably earlier than $40 \%$, a cubic-equation fit produces a quite narrow high-velocity peak; the consequence is similar to shortening saccadic duration because the extended right-hand tail of the velocity curve does not affect estimates of peak velocity except for saccades of very short duration-see Figure 2.) The two fitted equations for each data set in Figure 3, which peak at $40 \%$ and $50 \%$ of duration, obviously do not provide ideal matches with observation. In particular, the pattern of initial acceleration is poorly captured. Nevertheless, the general shape of real saccades is not seriously misrepresented at times surrounding peak velocity, which is the region of interest here; hence, the simulation study has emphasized that range of saccadic shapes. (In order to match the velocity profile of saccades appreciably larger than $20^{\circ}$ in excursion, greater skewness would be desirable, with peak velocity somewhat earlier than $40 \%$ of duration: See examples in Van Opstal \& Van Gisbergen, 1987, and Collewijn et al., 1988.)

Integration of a chosen velocity equation provides simulated values for eye position during a saccade. Such position curves can then be sampled at intervals of $16.66 \mathrm{msec}$ (the time between successive video pictures). Velocity is then derived from the 2-point difference in the eye-position data. The questions of interest here involve the relationship between sampled estimates of velocity and true peak velocity. Therefore, the velocity it-

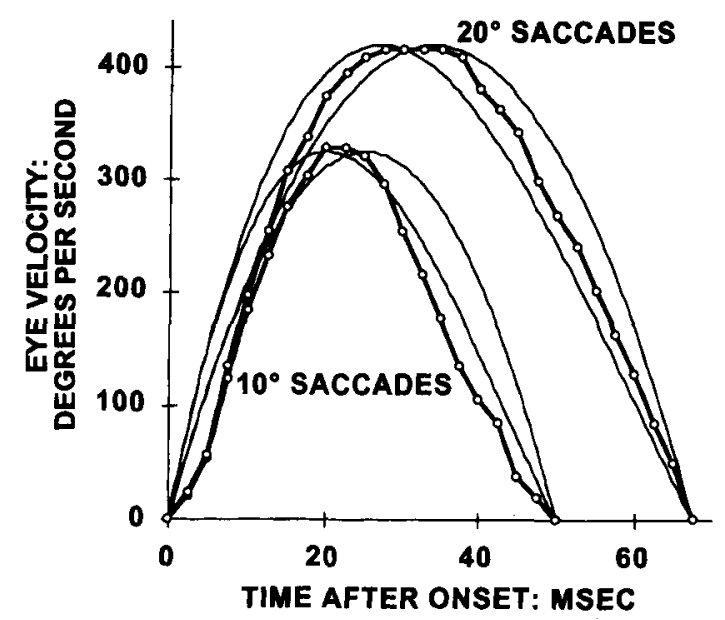

Figure 3. Empirical data for $10^{\circ}$ and $20^{\circ}$ horizontal saccades (open circles, connected by heavy lines: averages over 4 saccades each), based on $500-\mathrm{Hz}$ measurements made with scleral search coils; data derived from Figure 2 in Collewijn, Erkelens, and Steinman (1988) by superimposing a transparent grid on a muchenlarged photocopy of the continuous velocity curves. Both empirical data sets were accompanied by fitted cubic equations that peak at $40 \%$ or $50 \%$ of saccadic duration. self, in dimensions of deg/sec, need not be directly included in the calculations, as was done for Figure 3 . The two key aspects of the cubic equations used in the simulations then become the duration of the saccade in milliseconds; the skewness, as represented by the time at which peak velocity occurs (here expressed as a fraction of total duration). In addition, it is evident that the "phasing" (i.e., temporal interlacing) of video pictures relative to the timing of the saccade will play a major role in each resulting estimate of peak velocity. When a pair of simulated position measurements symmetrically straddles the time of true peak velocity, the velocity estimate will clearly differ from (and is likely to be greater than) that obtained when one of the two position measurements is made at the peak of the velocity curve. Three examples of this influence of phasing are shown in Figure 4, with first intrasaccadic measurement of position made at onset of the saccade, $6 \mathrm{msec}$ after onset, or $12 \mathrm{msec}$ after onset.

Extensive numerical simulations were undertaken that incorporated several saccadic durations between 30 and $100 \mathrm{msec}$ and peak timings of $40 \%$ and $50 \%$ of duration; all possible timings of the saccade relative to the measurements of position were included (rectangular distribution of first intrasaccadic pictures, ranging from 0 to $16 \mathrm{msec}$ after saccadic onset). The estimates of peak velocity resulting from some of those simulations are summarized in Figure 5, which illustrates both the downward biases of the average estimates and the trial-to-trial variability expected because of variations in temporal interlacing.

There are two noteworthy aspects of these simulation results. First, as saccades become smaller (and hence, have shorter durations), the downward bias in estimates of peak velocity increases, but even with saccades as brief as $50 \mathrm{msec}$ (excursions of about $10^{\circ}$, with only three position measurements usually made during the saccade itself), the average bias is considerably smaller than $10 \%$. Second, the variability to be expected among replicate estimates of peak velocity from identical saccades is remarkably small, with standard deviations due to adventitious differences in saccade timing being only $2 \%$ to $4 \%$ even for $50-\mathrm{msec}\left(10^{\circ}\right)$ saccades.

Although the precise values shown in Figure 5 depend upon use of a cubic equation to simulate saccadic velocity, very similar values would be expected from fitting realistic profiles of saccadic velocity by using other functions, such as a higher-order polynomial or the gamma distribution (which Van Opstal \& Van Gisbergen, 1987, found to give satisfactory fits over a broad range of data). The critical issue for present purposes is only the general shape of the velocity profile around time of midsaccade, for which the third-order polynomial seems satisfactory (Figure 3).

Data from the simulations for saccadic duration of 30 and 40 msec shown in Figure 5 demonstrate that the magnitudes of bias and variability due to the low sampling rate of video become increasingly severe problems. (Duration of $35 \mathrm{msec}$ corresponds to saccadic amplitude of about $5^{\circ}$.) Nevertheless, for saccades of intermediate to 


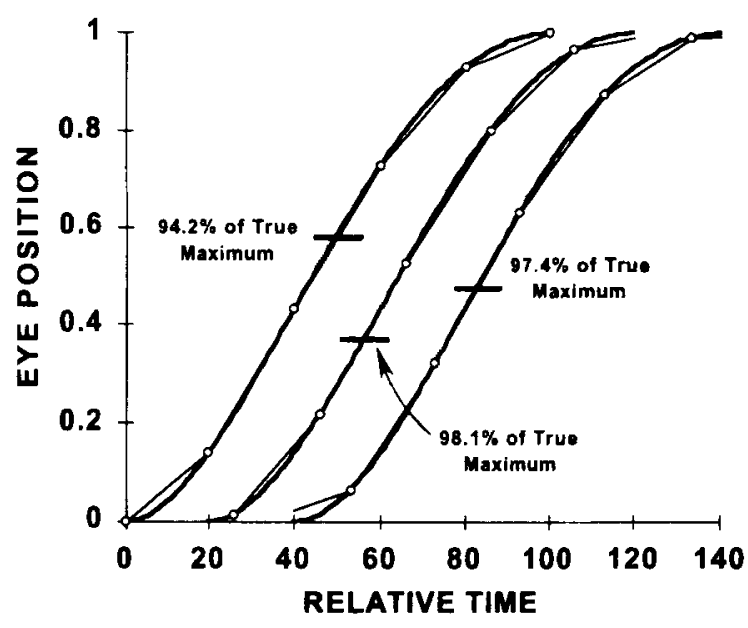

Figure 4. Schematic diagram illustrating video-estimated peak velocity as influenced by phase relationship between simulated saccade (smooth, heavy-line curve, based on saccadic duration of $80 \mathrm{msec}$ and peak velocity at $40 \%$ of duration) and timing of video measurements (open circles at intervals of $16 \mathrm{msec}$ ). Note that abscissa involves "relative time," with 100 units corresponding to the saccadic duration of $80 \mathrm{msec}$. First intrasaccadic video picture was simulated at 0,6 , or 12 msec after saccadic onset, respectively, for three illustrated curves, which have been displaced along the abscissa for clarity. Estimates of interpicture average velocity correspond to slopes of thin straight lines connecting open circles; segments of a given saccade during which maximum interpicture velocity occurred are indicated by short horizontal bars through position curve, with that velocity given as fraction of true peak velocity.

large excursion, like those in Figure 1, this simulation study indicates that the coarse temporal resolution of video recording may well be adequate for estimating "apparent peak saccadic velocity," as long as certain limitations are recognized, which can now be roughly quantified.

In order to examine the specifics of those limitations, consider again the data presented in Figure 1. The simulations described here confirm, of course, that the individual empirical estimates of peak velocity in Figure 1 are biased downward; Figure 5 indicates that on average, the magnitude of this bias is probably in the range between $3 \%$ and $8 \%$. Furthermore, because the expected downward bias in the estimates is greater for briefer (and hence, for smaller) saccades, the slopes of the regression lines illustrated in Figure 1 can be assumed to be somewhat steeper than would have been found had monitoring been undertaken with a higher sampling rate. Perhaps most interesting, however, is that the trial-to-trial variability in the empirical estimates of peak velocity, with a standard deviation of $14 \%$ to $17 \%$ around the corresponding regression lines in Figure 1, can be taken as being primarily due to genuine between-trial differences in performance. If position were measured without error, and if the saccades were otherwise fully comparable, with a uniquely defined relationship between peak velocity and saccadic excursion (as in the simulations), the coarse sampling rate of video recording would have been expected to leave residual variability in the estimates with standard deviations of well less than $5 \%$. As noted earlier, imprecision in the measurement of position can be expected to have contributed standard deviations of about $8.4 \%$ sec to the velocity estimates, or about $2 \%$ to $4 \%$ of the mean values. Even for the smaller saccades of Figure 1 , therefore, intrinsic variability in performance between trials would represent the primary source of the scatter in the data, contributing more than two thirds of the total variance. It would, of course, require highfrequency information from many high-precision recordings to determine whether that residual trial-to-trial variability primarily represents genuine differences between saccades in peak velocity for a given excursion; or perhaps it is due to something more subtle, such as cross-trial differences in duration and skewness of the velocity profiles (although major variability in skewness seems unlikely on the basis of the reproducibility of saccadic skewness in the examples presented by Van Opstal \& Van Gisbergen, 1987).

The interpretations reported here stand in stark contrast to the discouraging results reported by Harris et al. (1984), but there are conspicuous differences between the two approaches, which presumably can account for the discrepancy. Their recording system involved automated evaluation of distance between the center of a corneal reflection (first Purkinje image) and the pupillary margin, rather than direct measurement of limbus position relative to an electronically imposed reference line in the pictures. The Purkinje reflection may change its shape due to altered corneal orientation; the pupil is photographed with prismatic distortion through the cornea and can also change in actual size independently of eye orientation. Their video cameras apparently in-

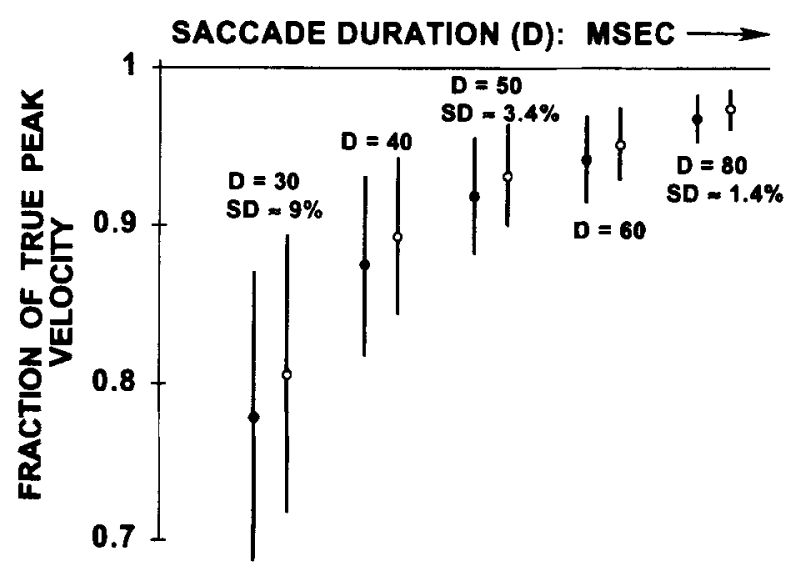

Figure 5. Summary of estimates of peak velocity of saccades relative to true peak velocity (average values bracketed by $\pm 1 S D$ ) from simulations of 60-Hz video recordings for saccades with durations $(D)$ of $30,40,50,60$, and 80 msec having peak velocities occurring at $40 \%$ (filled circles) or $50 \%$ (open circles) of saccadic duration. Calculations based on assumption that all phase relationships ("interlacings") between video framing and saccade timing are equally likely. Real saccades of $5^{\circ}, 10^{\circ}$, and $20^{\circ}$ excursion typically have durations of about 35,50 , and $80 \mathrm{msec}$, respectively (Collewijn, Erkelens, \& Steinman, 1988). 
corporated no equivalent of the very brief shutter speed available with a CCD camera, meaning that the intrasaccadic pictures were probably somewhat (if not severely) blurred. In addition, the nominal single-measurement precision of their system (manufacturer's claim, presumably for a stationary eye), was $0.5^{\circ}$, which is about fivefold poorer than the method used here. And finally, their theoretical evaluation involved Fourier analysis of the output obtained with an artificial eye that was mechanically rotated - with no demonstration that movement of the model eye adequately mimicked the behavior of a real eye.

\section{CAUTIONARY NOTES}

The experimental observations considered here (Figure 1) were obtained by manual analysis of recordings obtained with the equipment specified in the legend of Figure 1, equipment that is distinctly different from (and less expensive than) most commercially available video systems designed for eye-movement monitoring. The study described here demonstrates that the $60 \mathrm{-Hz}$ temporal resolution provided by this system (and many others) does not represent an insuperable handicap for estimation of peak saccadic velocity with reasonably good accuracy and precision. Although it has thus been demonstrated that equipment of the sort described here can do the job for larger sized saccades, each individual video system must be evaluated in terms of shutter speed, image magnification, measurement precision ("noise" levels), and playback speed in order to decide whether similar performance can be expected. For example, many consumergrade VCRs designed for home use can provide only 30 $\mathrm{Hz}$ single-frame playback, rather than the $60-\mathrm{Hz}$ singlefield playback considered here; $30-\mathrm{Hz}$ measurements would present much more severe problems.

In some kinds of eye-movement research, it is important to distinguish between saccades and other, slower velocity, eye movements, or to have precise estimates of exactly when a saccade begins; for those purposes, it is customary to use a preselected velocity-of-movement threshold to define saccade onset. Clearly this sort of criterion cannot be applied with meaningful precision to $60-\mathrm{Hz}$ video recordings, nor would it be possible to obtain reliable profiles of the full time course of velocity or to reliably estimate ocular acceleration during a saccade, based on $60-\mathrm{Hz}$ video. For those problems, far better temporal resolution is essential.

\section{CONCLUSIONS}

These simulations indicate that although the low temporal resolution of video recording introduces both bias and adventitious variation into derived estimates of peak saccadic velocity, those sources of error may well be tolerable for saccades with excursions greater than about $10^{\circ}$, given a suitable video recording system. Certainly those potential problems need not seriously compromise the ability to detect treatment-specific differences of, say, $10 \%$ in peak velocity (Figure 1 ). With as large a data set as available there, even a difference of $3 \%$ in average velocity between abducting and adducting saccades would have been statistically significant at the .05 level. That conclusion in fact follows from calculations based directly on the data themselves and not from the simulation study; the simulations, however, serve to demonstrate that such data can readily be reconciled with the video monitoring method from which they were derived. Given suitable equipment, video monitoring can thus provide a simple, noninvasive, and relatively inexpensive method for evaluations of peak saccadic velocity with accuracy and precision that may be adequate for a variety of applications, provided that the saccades are, say, $10^{\circ}$ or larger in excursion.

\section{REFERENCES}

Clarke, A. H. (1994). Image processing techniques for the measurement of eye movement. In J. Ygge \& G. Lennerstrand (Eds.), Eye movements in reading (pp. 21-38). Oxford: Pergamon.

Collewijn, H., Erkelens, C. J., \& Steinman, R. M. (1988). Binocular co-ordination of human horizontal saccadic eye movements. Journal of Physiology, 404, 157-182.

ENRIGHT, J. T. (1984). Changes in vergence mediated by saccades. Journal of Physiology, 350, 9-31.

Harris, C. M., Abramov, I., \& Hainline, L. (1984). Instrument considerations in measuring fast eye movements. Behavior Research Methods, Instruments, \& Computers, 16, 341-350.

HASLWANTER, T., \& MOORE, S. (1995). A theoretical analysis of three dimensional eye position measurement using polar cross correlation. IEEE Transactions on Biomedical Engineering, 42, 1053-1061.

Moore, S., Haslwanter, T., Curthoys, I., \& Smith, S. (1996). A geometric basis for measurement of three dimensional eye position using image processing. Vision Research, 36, 445-459.

Van Opstal, A. J., \& Van Gisbergen, J. A. M. (1987). Skewness of saccadic velocity profiles: A unifying parameter for normal and slow saccades. Vision Research, 27, 731-745.

(Manuscript received May 16, 1996; revision accepted for publication October $7,1996$. 